\title{
Chandra, MDM, Swift, and NuSTAR Observations Confirming the SFXT Nature of AX J1949.8+2534
}

\section{Citation}

Hare, Jeremy, Jules P. Halpern, Maïca Clavel, Jonathan E. Grindlay, Farid Rahoui, and John A. Tomsick. 2019. Chandra, MDM, Swift, and NuSTAR Observations Confirming the SFXT Nature of AX J1949.8 2534. The Astrophysical Journal 878, no. 1: 8.

\section{Permanent link}

http://nrs.harvard.edu/urn-3:HUL.InstRepos:42389120

\section{Terms of Use}

This article was downloaded from Harvard University's DASH repository, and is made available under the terms and conditions applicable to Other Posted Material, as set forth at http:// nrs.harvard.edu/urn-3:HUL.InstRepos:dash.current.terms-of-use\#LAA

\section{Share Your Story}

The Harvard community has made this article openly available.

Please share how this access benefits you. Submit a story.

Accessibility 


\title{
Chandra, MDM, Swift, and NuSTAR Observations Confirming the SFXT Nature of AX $\mathrm{J} 1949.8+2534$
}

\author{
Jeremy Hare $^{1}$ (D), Jules P. Halpern ${ }^{2}$, Maïca Clavel ${ }^{3}$ (D) Jonathan E. Grindlay $^{4}$, Farid Rahoui ${ }^{5}$ (D), and John A. Tomsick ${ }^{1}$ (D) \\ ${ }^{1}$ Space Sciences Laboratory, 7 Gauss Way, University of California, Berkeley, CA 94720-7450, USA; jhare@ berkeley.edu \\ ${ }^{2}$ Department of Astronomy, Columbia University, 550 West 120th Street, New York, NY 10027, USA \\ ${ }^{3}$ Universite Grenoble Alpes, CNRS, IPAG, F-38000 Grenoble, France \\ ${ }^{4}$ Harvard-Smithsonian Center for Astrophysics, 60 Garden Street, Cambridge, MA 02138, USA \\ ${ }^{5}$ Department of Astronomy, Harvard University, 60 Garden Street, Cambridge, MA 02138, USA \\ Received 2019 February 12; revised 2019 April 12; accepted 2019 April 19; published 2019 June 7
}

\begin{abstract}
AX J1949.8+2534 is a candidate supergiant fast X-ray transient (SFXT) observed in outburst by the International Gamma-ray Astrophysics Laboratory (IGR J19498+2534). We report on the results of six Neil Gehrels SwiftXRT, one Chandra, and one Nuclear Spectroscopic Telescope Array observation of the source. We find evidence of rapid X-ray variability on a few kilosecond timescales. Fortunately, Chandra observed the source in a relatively bright state, allowing us to confidently identify the optical/NIR counterpart of the source. We also obtained an optical spectrum of this counterpart, which shows an $\mathrm{H} \alpha$ emission line and $\mathrm{He} \mathrm{I}$ absorption features. The photometry and spectrum of the source allow us to constrain its distance, $\sim 7-8 \mathrm{kpc}$, and reddening, $A_{V}=8.5-9.5$. We find that the star is likely an early B-type Ia supergiant, confirming that AX J1949.8+2534 is indeed an SFXT.
\end{abstract}

Key words: supergiants - X-rays: binaries - X-rays: individual (IGR J19498+2534)

Supporting material: data behind figure

\section{Introduction}

The International Gamma-ray Astrophysics Laboratory (INTEGRAL) mission (Winkler et al. 2003) has been observing the hard X-ray sky for more than 16 yr. During this time it has uncovered a large number of new Be high-mass X-ray binaries (BeHMXBs), while also dramatically increasing the population of supergiant HMXBs (sgHMXBs; see, e.g., Figure 4 in Walter et al. 2015). Additionally, INTEGRAL has unveiled new subclasses of sgHMXBs: supergiant fast X-ray transients (SFXTs; Sguera et al. 2005; Negueruela et al. 2006b) and obscured supergiant HMXBs (e.g., IGR J16318-4848; Matt \& Guainazzi 2003). There are also several subclasses of SFXTs, which exhibit different dynamical ranges in their X-ray luminosities, from $\sim 10^{2}$ to $10^{5}$ and different flare timescales (see, e.g., Table 2 in Chaty 2011; Walter et al. 2015). Among all of these systems there are also a number of "intermediate" HMXBs, that do not entirely fit into these categories and have properties that lie in between the classes (see, e.g., Sidoli \& Paizis 2018). In order to understand the different physical processes responsible for producing the emission observed in these subclasses and how they depend on the binary parameters (e.g., orbital period, eccentricity), it is important to increase the number of known members by identifying them through their $\mathrm{X}$-ray and optical properties.

Prior the to launch of INTEGRAL, BeHMXBs dominated the population of known HMXBs (Walter et al. 2015). BeHMXBs consist of a compact object (CO), typically a neutron star (NS), and a nonsupergiant Be spectral type companion, generally having longer orbital periods, $P_{\text {orb }} \gtrsim 20$ days, than sgHMXBs (see Reig 2011 for a review). The Be companions are rapidly rotating, have $\mathrm{H} \alpha$ emission lines, and have an infrared (IR) excess compared to B stars (Porter \& Rivinius 2003). The Ho emission and IR excess come from a dense equatorial decretion disk rotating at or near Keplerian velocity, likely formed due to the fast rotation of the star (see Rivinius et al. 2013 for a review). These systems can be both persistent, with $\mathrm{X}$-ray luminosities on the order of $L_{\mathrm{X}} \approx 10^{34}-10^{35} \mathrm{erg} \mathrm{s}^{-1}$, or transient (Reig 2011). The transients become X-ray bright when the NS accretes material as it passes near (or through) the Be star's decretion disk. These systems exhibit two types of $\mathrm{X}$-ray flares. Type I flares occur periodically or quasiperiodically when the NS passes through periastron, increasing the X-ray flux by an order of magnitude, and lasting a fraction of the orbital period $\left(\sim 0.2 \times P_{\text {orb }} \approx 1-10\right.$ days; Reig 2011). Type II flares can occur at any orbital phase, reaching Eddington luminosity, and lasting a large fraction of an orbital period ( $\sim 10-100$ days; Reig 2011).

The sgHMXBs are composed of a $\mathrm{CO}$ and a supergiant $\mathrm{O}$ or B spectral type companion, typically with shorter orbital periods, $P_{\text {orb }} \lesssim 10$ days, than BeHMXBs (Reig 2011; Walter et al. 2015). If the CO orbits close enough to the companion star, it can accrete via Roche lobe overflow, reaching X-ray luminosities up to $L_{\mathrm{X}} \sim 10^{38} \mathrm{erg} \mathrm{s}^{-1}$ during an accretion episode (Chaty et al. 2008; Chaty 2015). For longer period systems, the CO accretes from the fast $\left(\sim 1000 \mathrm{~km} \mathrm{~s}^{-1}\right)$ radiative wind of the supergiant companion, leading to persistent X-ray luminosities of $L_{\mathrm{X}} \sim 10^{35}-10^{36} \mathrm{erg} \mathrm{s}^{-1}$ (Chaty et al. 2008). These wind-fed systems are also often highly obscured $\left(N_{\mathrm{H}} \gtrsim 10^{23} \mathrm{~cm}^{-2}\right)$ by the wind of the companion, and in some cases, by an envelope of gas and dust around the entire binary system (see, e.g., IGR J16318-4848; Walter et al. 2003; Filliatre \& Chaty 2004). The supergiant stars in these systems also exhibit an $\mathrm{H} \alpha$ emission line due to their winds, which is often variable in shape and intensity and can have a P-Cygni profile (see e.g., Vela X-1, IGR J11215-5952; Barziv et al. 2001; Lorenzo et al. 2010).

INTEGRAL's wide field of view has enabled great progress in the study of HMXBs by discovering many SFXTs (see, e.g., Negueruela et al. 2006b, or Sidoli 2013, 2017 for reviews). Unlike the typical sgHMXBs, SFXTs exhibit much lower quiescent X-ray luminosities (as low as $L_{\mathrm{X}} \sim 10^{32} \mathrm{erg} \mathrm{s}^{-1}$ ) with 
highly energetic $\left(L_{\mathrm{X}} \sim 10^{36}-10^{38} \mathrm{erg} \mathrm{s}^{-1}\right)$ X-ray flares lasting $\sim 100-10,000 \mathrm{~s}$ (see, e.g., in't Zand 2005; Romano et al. 2014, 2015). INTEGRAL has also uncovered several systems, with flare to quiescent X-ray luminosity ratios of $10^{2}-10^{3}$, lasting a few hours to days (see, e.g., IGR J17354-3255; Sguera et al. 2011; Ducci et al. 2013). These systems have larger variability than seen in classic sgHMXB systems, but longer variability timescales than in SFXTs, and therefore have been called intermediate SFXTs (Chaty 2011; Sguera et al. 2011).

Several models have been put forward to describe the flaring phenomenon observed in SFXTs. One possibility is that the flares are caused by the accretion of an inhomogeneous clumpy wind, produced by the high-mass stellar companion, onto the CO (see, e.g., in't Zand 2005; Negueruela et al. 2006b; Walter \& Zurita Heras 2007; Ducci et al. 2009; Bozzo et al. 2016). However, given the low-quiescent luminosities of some SFXTs, an additional mechanism for inhibiting the accretion onto the $\mathrm{CO}$ is likely necessary (e.g., magnetic gating or subsonic accretion; Grebenev \& Sunyaev 2007; Bozzo et al. 2008; Shakura et al. 2012).

The source AX J1949.8+2534 was discovered by the $A S C A$ Galactic plane survey, having an absorbed flux of $6 \times$ $10^{-12} \mathrm{erg} \mathrm{cm}^{-2} \mathrm{~s}^{-1}$ in the $2-10 \mathrm{keV}$ band (Sugizaki et al. 2001). AX J1949.8+2534 (AX J1949 hereafter) was then detected by INTEGRAL for the first time in the hard X-ray band during two short flaring periods in 2015/2016 (Sguera et al. $2015){ }^{6}$ The first flaring episode lasted $\lesssim 1.5$ days and reached a peak flux $F_{\mathrm{X}}=1.1 \times 10^{-10} \mathrm{erg} \mathrm{cm}^{-2} \mathrm{~s}^{-1}$ in the $22-60 \mathrm{keV}$ band, while the second flaring episode lasted $\sim 4$ days with a similar peak flux $F_{\mathrm{X}}=1.0 \times 10^{-10} \mathrm{erg} \mathrm{cm}^{-2} \mathrm{~s}^{-1}$ in the 22-60 keV band (Sguera et al. 2017). However, during the second flaring episode shorter timescale variability of $\sim 2-8 \mathrm{ks}$ was detected, reaching a peak flux of $2 \times 10^{-9} \mathrm{erg} \mathrm{cm}^{-2} \mathrm{~s}^{-1}$ in the $200 \mathrm{~s}$ binned light curve (Sguera et al. 2017). The dynamic range of the flare to quiescent $\mathrm{X}$-ray luminosities in the $20-40 \mathrm{keV}$ band is $\gtrsim 625$ (Sguera et al. 2017).

In soft X-rays, Sguera et al. (2017) also reported on a Neil Gehrels Swift-XRT observation of the source, which was detected with an absorbed flux of $1.8 \times 10^{-12} \mathrm{erg} \mathrm{cm}^{-2} \mathrm{~s}^{-1}$. This observation also provided a more accurate position for the source. However, there were two bright potential NIR counterparts within the $95 \%$ Swift positional uncertainty. Based on photometry, Sguera et al. (2017) classified these two bright NIR sources as B0V and B0.5Ia spectral type stars, respectively, leading to the conclusion that this source was either a BeHMXB or SFXT type source. The bright flares observed from AX J1949 disfavored the BeHXMB scenario; therefore, Sguera et al. (2017) favored the SFXT interpretation.

In this paper we report on Neil Gehrels Swift-XRT, Chandra, and Nuclear Spectroscopic Telescope Array (NuSTAR) legacy observations of the SFXT candidate AX J1949. These observations are part of an ongoing program to identify the nature of unidentified INTEGRAL sources by characterizing their broadband $(0.3-79.0 \mathrm{keV}) \mathrm{X}$-ray spectrum. Additionally, we use the precise X-ray localization to identify the multiwavelength counterpart for optical spectroscopic follow-up, which is also reported here.

\footnotetext{
6 The source is also referred to as IGR J19498+2534 in the INTEGRAL catalog of Krivonos et al. (2017).
}

Table 1

Swift-XRT Observations and Signal-to-noise Ratio of AX J1949

\begin{tabular}{lccccc}
\hline \hline$\#$ & ObsID & $\begin{array}{c}\text { Offset }^{\mathrm{a}} \\
\left(\operatorname{arcmin}^{2}\right)\end{array}$ & $\begin{array}{c}\text { Start Time } \\
(\mathrm{MJD})\end{array}$ & $\begin{array}{c}\text { Exp. } \\
(\mathrm{s})\end{array}$ & $\mathrm{S} / \mathrm{N}$ \\
\hline S0 $^{\mathrm{b}}$ & 00034497001 & 2.422 & 57503.446 & 2932 & 5.3 \\
S1 & 00010382001 & 2.997 & 58159.464 & 4910 & 5.5 \\
S2 & 00010382002 & 2.240 & 58166.446 & 3369 & 5.4 \\
S3 & 00010382003 & 2.441 & 58170.346 & 1568 & 2.2 \\
S4 & 00010382004 & 2.938 & 58173.336 & 4662 & 2.6 \\
S5 & 00010382005 & 0.424 & 58179.584 & 5482 & 4.5 \\
\hline
\end{tabular}

Note.

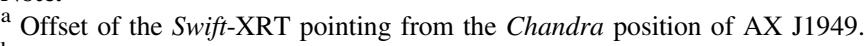

b This observation was originally reported on by Sguera et al. (2017).

\section{Observations and Data Reduction}

\subsection{Neil Gehrels Swift X-Ray Observatory}

The Neil Gehrels Swift satellite's (Gehrels et al. 2004) X-ray telescope (XRT; Burrows et al. 2005) has observed AX J1949 a total of six times, once in 2016 and five more times in a span of $\sim 20$ days in 2018. The 2016 observation was originally reported on by Sguera et al. (2017) (although for consistency we reanalyze it here), while the five observations from 2018 are reported on for the first time in this paper. The details of each Swift-XRT observation can be found in Table 1 . We refer to each observation by the associated number shown in Table 1. The data were reduced using HEASOFT version 6.25 and the 2018 July version of the Swift-XRT Calibration Database (CALDB). The Swift-XRT instrument was operated in photon counting mode and the event lists were made using the xrtpipeline task. To estimate the count rates, source significances, and upper limits we used the uplimit and source statistics tool (sosta) in the XIMAGE package (version 4.5.1). The count rates and upper limits were also corrected for vignetting, dead time, the point-spread function (PSF), and bad pixels/columns that overlap with the source region.

We extracted the source spectrum for each Swift observation in which the source was detected with a signal-to-noise ratio $(\mathrm{S} / \mathrm{N})>3$ using XSELECT (version 2.4). A new ancillary response file was constructed with the xrtmkarf task using the exposure map created by the xrtpipeline task, and the corresponding response file was retrieved from the CALDB. The observed count rates (with no corrections applied) of the source in these observations were between $\sim(8$ and 12) $\times 10^{-3}$ cts $^{-1}$, so we used circular extraction regions with radii of 12 or 15 pixels depending on the observed count rate (see Table 1 in Evans et al. 2009). Background spectra were extracted from a source-free annulus centered on AX J1949. The Swift spectra were grouped to have at least one count per bin and fit using Cash statistics (C-stat; Cash 1979).

In this paper, all spectra are fit using XSPEC (version 12.10.1; Arnaud 1996). We used the Tuebingen-Boulder ISM absorption model (tbabs) with the solar abundances of Wilms et al. (2000). All uncertainties reported in this paper (unless otherwise noted) are $1 \sigma$.

\subsection{Chandra X-Ray Observatory}

We observed AX J1949 using the Chandra Advanced CCD Imaging Spectrometer (ACIS; Garmire et al. 2003) on 2018 February 25 (MJD 58174.547; obsID 20197) for $4.81 \mathrm{ks}$. The 
source was observed by the front-illuminated ACIS-S3 chip in timed exposure mode and the data were telemetered using the "faint" format. A 1/4 subarray was used to reduce the frame time to $0.8 \mathrm{~s}$, ensuring that the pileup remained $<3 \%$ throughout the observation. All Chandra data analysis was performed using the Chandra Interactive Analysis of Observations (CIAO) software version 4.10 and CALDB version 4.8.1. The event file was reprocessed using the CIAO tool chandra_repro prior to analysis.

To locate all sources in the field of view, the CIAO tool wavdetect was run on the $0.5-8 \mathrm{keV}$ band image. ${ }^{7}$ Only one source, the counterpart of AX J1949, was detected at the position R.A. $=297^{\circ} .48099$, decl. $=25^{\circ} .56639$ with a statistical $95 \%$ confidence positional uncertainty of 0 ". 13 estimated using the empirical relationship (i.e., Equation (12)) from Kim et al. (2007). We were unable to correct for any systematic uncertainty in the absolute astrometry because only one X-ray source was detected. Therefore, we adopt the overall $90 \%$ Chandra systematic uncertainty of $0.8^{8}$ and convert it to the $95 \%$ uncertainty by multiplying by $2.0 / 1.7$. The statistical and systematic errors were then added in quadrature, giving a $95 \%$ confidence positional uncertainty radius of $0 . " 95$ for the source.

The Chandra energy spectrum of AX J1949 was extracted from a circular region centered on the source and having a radius of $2^{\prime \prime}$, enclosing $\sim 95 \%$ of the PSF at $1.5 \mathrm{keV},{ }^{9}$ and containing 260 net counts. The background spectrum was extracted from a source-free annulus centered on the source. Given the small number of counts, we fit the Chandra spectrum using C-stat (Cash 1979).

We have also extracted the Chandra light curves using a number of different binnings to search for spin and orbital periods in the data. Prior to extraction, the event times were corrected to the solar system barycenter using the CIAO tool axbary.

\subsection{NuSTAR}

NuSTAR (Harrison et al. 2013) observed AX J1949 on 2018 February 24 (MJD 58173.070; obsID 30401002002) for $45 \mathrm{ks}$. We reduced the data using the NUSTAR Data Analysis Software (NuSTARDAS) version 1.8.0 with CALDB version 20181022. Additionally, we filtered the data for background flares caused by $N U S T A R$ 's passage through the South Atlantic Anomaly using the options saacalc $=2$, saamode $=$ optimized, and tentacle $=$ yes, which reduced the total exposure time to $43 \mathrm{ks}$.

The source's energy spectra from the FPMA and FPMB detectors were extracted from circular regions with radii of $45^{\prime \prime}$ centered on the source position. The background spectra were extracted from source-free regions away from AX J1949, but on the same detector chip. The NUSTAR spectra were grouped to have at least one count per bin. NuSTAR light curves were also extracted from both the FPMA and FPMB detectors in the 3-20 keV energy range using a number of different bin sizes (i.e., 100, $500 \mathrm{~s}, 1,5 \mathrm{ks}$ ). All NuSTAR light curves plotted in this paper show the averaged (over the FPMA and FPMB detectors) net count rate.

\footnotetext{
7 We ran wavdetect using the exposure map and PSF map produced by the CIAO tools fluximage and mks fmap, respectively.

8 http://cxc.harvard.edu/cal/ASPECT/celmon/

9 See Chapter 4, Figure 4.6 at http: / cxc.harvard.edu/proposer/POG/html/.
}

\subsection{MDM Spectroscopy}

The accurate position of the X-ray counterpart to AX J1949 provided by Chandra has allowed us to identify the optical/ NIR counterpart to the source. The optical/NIR counterpart is the brightest source (see Table 3) considered by Sguera et al. (2017) as a potential counterpart (i.e., their source 5). This source has a Gaia position R.A. $=297^{\circ} .480949745(9)$ and decl. $=25^{\circ} .56659555(1)$, which is $\sim 0.76$ offset from the Chandra source position and within the $2 \sigma$ Chandra positional uncertainty.

On 2018 October 18, a 600 s spectrum was obtained with the Ohio State Multi-Object Spectrograph on the $2.4 \mathrm{~m}$ Hiltner telescope of the MDM Observatory on Kitt Peak, Arizona. A 1 ". 2 wide slit and a volume-phase holographic grism provided a dispersion of $0.72 \AA$ pixel $^{-1}$ and a resolution of $\approx 3 \AA$ over the wavelength range $3965-6878 \AA$. The reduced spectrum is shown in Figure 1, where it can be seen that flux is not well detected below $4900 \AA$ due to the large extinction to the star. Although a standard star was used for flux calibration, the narrow slit and partly cloudy conditions are not conducive to absolute spectrophotometry. Therefore, as a last step we have scaled the flux to match the $V$ magnitude from Table 3.

\section{Results}

\subsection{X-Ray Spectroscopy}

AX J1949 is a variable X-ray source (see Figure 2), but, fortunately, Chandra observed it during a relatively bright state, allowing for the most constraining X-ray spectrum of all of the observations reported here. We fit the Chandra spectrum in the $0.5-8 \mathrm{keV}$ range with two models, an absorbed power-law model and an absorbed blackbody model (see Figure 3 ). The best-fit power-law model has a hydrogen absorption column density $N_{\mathrm{H}}=7.5_{-1.4}^{+1.6} \times 10^{22} \mathrm{~cm}^{-2}$, photon index $\Gamma=1.4 \pm$ 0.4 , and absorbed flux $(2.0 \pm 0.3) \times 10^{-12} \mathrm{erg} \mathrm{cm}^{-2} \mathrm{~s}^{-1}$ (in the $0.3-10 \mathrm{keV}$ band) with a C-stat of 121.5 for 182 d.o.f. On the other hand, the best-fit blackbody model has hydrogen absorption column density $N_{\mathrm{H}}=(4.7 \pm 1) \times 10^{22} \mathrm{~cm}^{-2}$, temperature $k T=1.5 \pm 0.2 \mathrm{keV}$, radius $r_{\mathrm{BB}}=150_{-20}^{+30} d_{7 \mathrm{kpc}} \mathrm{m}$, and absorbed flux $(1.6 \pm 0.2) \times 10^{-12} \mathrm{erg} \mathrm{cm}^{-2} \mathrm{~s}^{-1}$ (in the $0.3-10 \mathrm{keV}$ band $)$, where $d_{7 \mathrm{kpc}}=d /(7 \mathrm{kpc})$ is the assumed distance to the source (see Section 4.1), with a C-stat of 119.3 for 182 d.o.f. In either case, the photon index, or the blackbody temperature and emitting radius, are consistent with those observed in SFXTs (see, e.g., Table 4 in Romano et al. 2009). Therefore, because both models fit the data about equally well, we cannot distinguish between them.

We extracted the Swift-XRT spectra for all observations where the source was detected with an $\mathrm{S} / \mathrm{N}>3$ (i.e., S0, S1, $\mathrm{S} 2$, and S5). The source is not bright enough in any of these single observations to fit a constraining spectrum. Therefore, we jointly fit the Chandra spectrum with the four Swift-XRT spectra, tying together the hydrogen absorbing column, but leaving the photon index and normalization free to vary for each spectrum. The best-fit parameters can be seen in Table 2 . There is marginal evidence $(\sim 2 \sigma)$ of spectral softening in the spectrum of S2, which could also be due to a variable hydrogen absorbing column as is often seen in SFXTs (see, e.g., Sidoli et al. 2009; Bozzo et al. 2016; Goossens et al. 2019). We have also fit the spectra with an absorbed power-law model after tying together the $N_{\mathrm{H}}$ and photon index before fitting. When this is done, the best-fitting spectrum has a hydrogen absorption 


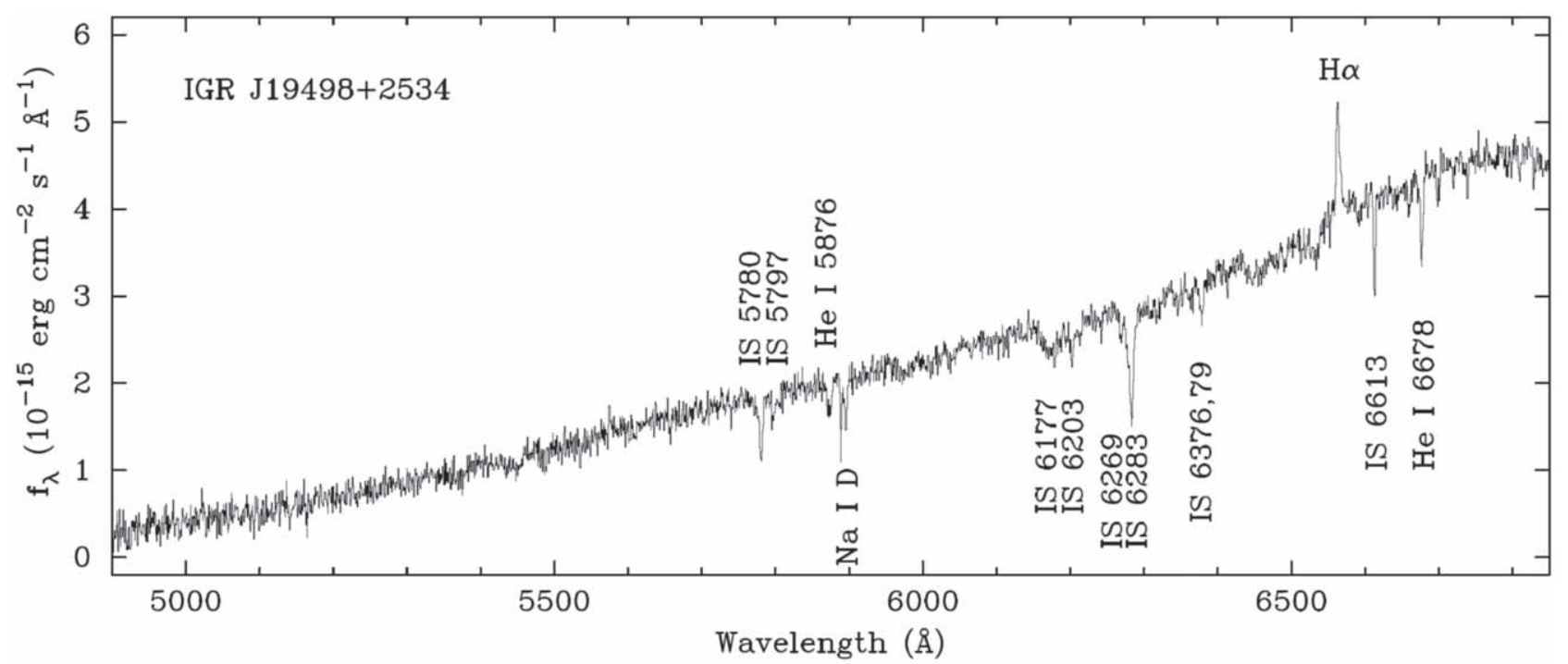

Figure 1. MDM $2.4 \mathrm{~m}$ optical spectrum of AX J1949 showing H $\alpha$ emission and He I absorption. The other spectral absorption features are diffuse interstellar bands caused by the large reddening toward the source $\left(A_{V}=8.5-9.5\right.$, see Section 4.1). The data used to create this figure are available.

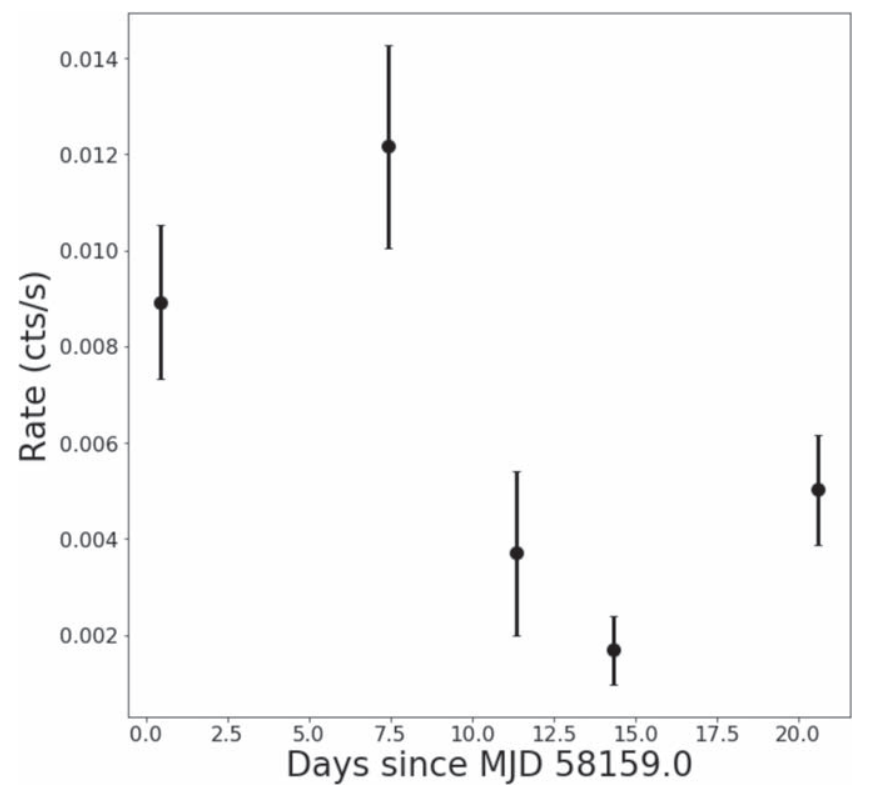

Figure 2. Swift-XRT net count rate light curve of AX J1949 during the $\sim 20$ day observing period in 2018, which included observations S1 through S5 (see Table 1).

column density $N_{\mathrm{H}}=5.6_{-1.0}^{+1.1} \times 10^{22} \mathrm{~cm}^{-2}$ and photon index $\Gamma=1.2 \pm 0.3$ with a C-stat of 275.0 for 295 d.o.f.

Unfortunately, AX J1949 was simultaneously observed with NuSTAR during the S4 Swift-XRT observation, when the source was at its faintest flux level (see Figure 4). Although the source was still jointly detected (i.e., FPMA+FPMB) with a significance of $5.3 \sigma$ and $5.7 \sigma$ (in the $3-79 \mathrm{keV}$ and $3-20 \mathrm{keV}$ energy bands, respectively), there were too few counts to constrain its spectrum. However, we extracted the $3 \sigma$ upperlimit flux in the 22-60 keV energy range for later comparison to INTEGRAL. To do this, we calculated the $3 \sigma$ upper limit on the net count rate of the source in the 22-60 keV energy range $\left(0.0011 \mathrm{cts} \mathrm{s}^{-1}\right)$. SFXTs often show a cutoff in their spectra between $\sim 5$ and $20 \mathrm{keV}$ (see e.g., Romano et al. 2011; Sidoli et al. 2017) so we use a power-law model with the best-fit

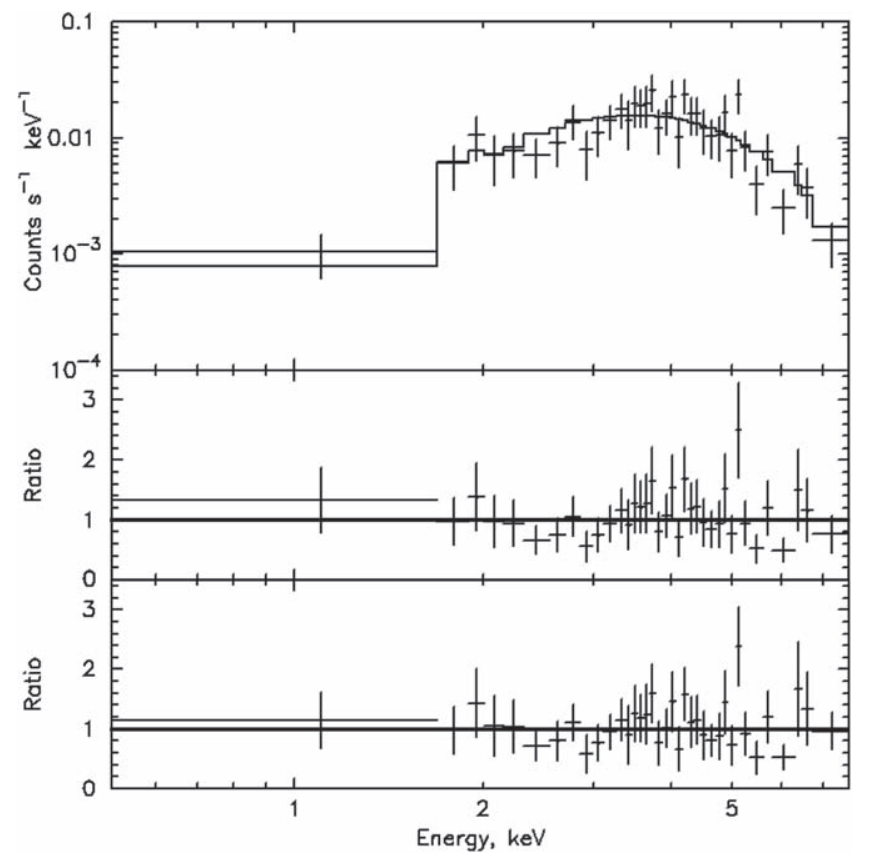

Figure 3. Chandra $0.5-8 \mathrm{keV}$ spectrum with best-fit power-law model (see Section 3.1 for best-fit parameters). The middle panel shows the ratio of the data to the power-law model, while the bottom panel shows the ratio of the data to the blackbody model. The spectra were binned for visualization purposes only.

absorption $\left(N_{\mathrm{H}}=5.6 \times 10^{22} \mathrm{~cm}^{-2}\right)$ and a softer photon index $(\Gamma=2.5)$ that is typical for SFXTs detected in hard X-rays by INTEGRAL (see, e.g., Sguera et al. 2008, 2011) to convert the count rate to flux. The $3 \sigma$ upper limit on the flux in the $22-60 \mathrm{keV}$ energy range is $F=5.8 \times 10^{-13} \mathrm{erg} \mathrm{cm}^{-2} \mathrm{~s}^{-1}$.

\subsection{X-Ray Variability and Timing}

The fluxes of the source are not constrained if the photon index is left free when jointly fitting the Chandra and SwiftXRT spectra. Therefore, to extract the fluxes from the Swift and Chandra observations we use the jointly fit model (i.e., $\Gamma=1.2$ 


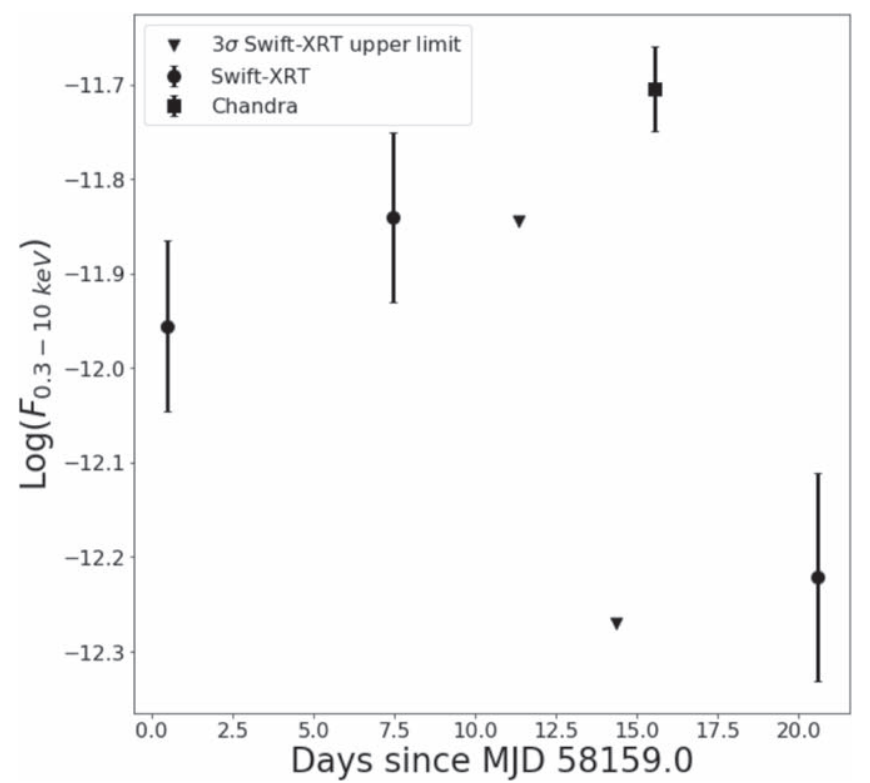

Figure 4. Fluxes of AX J1949 in the $0.3-10 \mathrm{keV}$ energy range from the jointly fit Swift-XRT and Chandra spectra (see Section 3.1). The points show the fluxes from the different observations (circles for Swift-XRT and squares for Chandra), while the triangles show the $3 \sigma$ upper limit of the source's flux when it was not detected in the Swift-XRT observations. The flux for observation S0 is excluded. The NuSTAR observation was concurrent with the S4 observation (i.e., the second triangle), when the flux of the source was at its minimum.

Table 2

Photon Indices for the Simultaneous Best-fit Absorbed Power-law Model

\begin{tabular}{lcccc}
\hline \hline Obs. & $\begin{array}{c}N_{\mathrm{H}} \\
\left(10^{22} \mathrm{~cm}^{-2}\right)\end{array}$ & $\Gamma$ & C-stat & d.o.f. \\
\hline Chandra & $5.6_{-1.0}^{+1.1}$ & $1.0 \pm 0.3$ & 243.4 & 286 \\
S0 & $5.6^{\mathrm{a}}$ & $1.0 \pm 0.5$ & $\cdots$ & $\cdots$ \\
S1 & $5.6^{\mathrm{a}}$ & $1.5 \pm 0.6$ & $\cdots$ & $\cdots$ \\
S2 & $5.6^{\mathrm{a}}$ & $2.5_{-0.5}^{+0.6}$ & $\cdots$ & $\cdots$ \\
S5 & $5.6^{\mathrm{a}}$ & $2.2 \pm 0.7$ & $\cdots$ & $\cdots$ \\
\hline
\end{tabular}

Note.

${ }^{\text {a }}$ The hydrogen absorption column density $\left(N_{\mathrm{H}}\right)$ values were tied together for the fit reported in this table.

and $N_{\mathrm{H}}=5.6 \times 10^{22} \mathrm{~cm}^{-2}$; see Section 3.1). The fluxes derived from these fits can be seen in Figure 4. Additionally, we assumed the same best-fit power-law model when converting the Swift $3 \sigma$ upper-limit count rates to fluxes. The flux from observation $\mathrm{S} 0$ is not shown in this figure but is

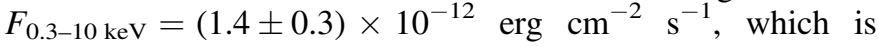
consistent within $2 \sigma$ of the value reported by Sguera et al. (2017).

We have also searched for shorter timescale variability of AX J1949 in the individual Chandra and NuSTAR observations. The $1 \mathrm{ks}$ binned net count rate light curve from the Chandra observation is shown in Figure 5. The light curve clearly shows that the source brightness decreased throughout the observation on a several kilosecond timescale. No significant flaring behavior was found in the Chandra light curves with smaller binnings. We also searched for any periodic signal in the Chandra data using the $Z_{1}^{2}$ (Buccheri et al. 1983), but no significant periodicity was found.

There are also indications of variability in the $3-20 \mathrm{keV}$ NuSTAR light curve. Notably, the 5 ks binned net light curve

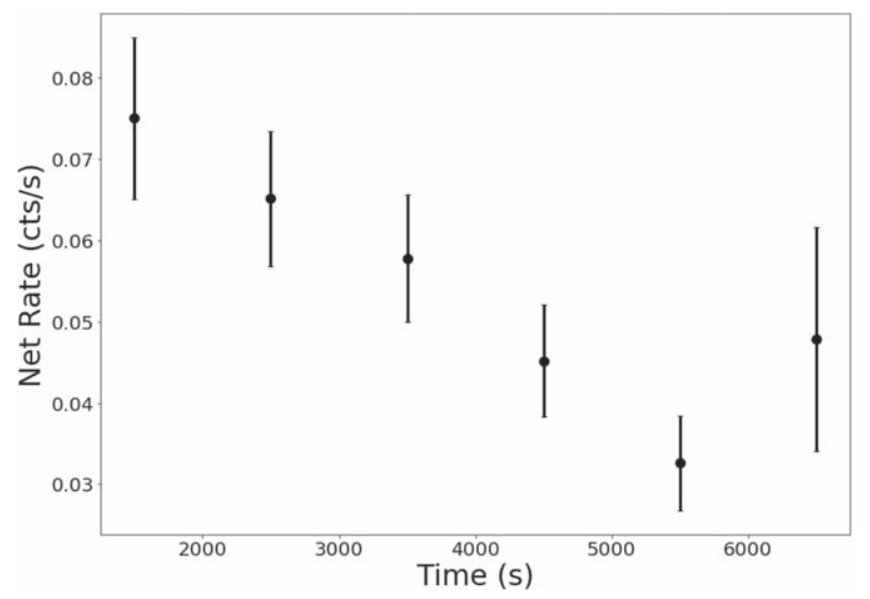

Figure 5. Chandra $0.5-8 \mathrm{keV}$ net count rate during the $4.81 \mathrm{ks}$ observation with a $1 \mathrm{ks}$ binning. The source was variable on $\sim 1 \mathrm{ks}$ timescales during this observation.

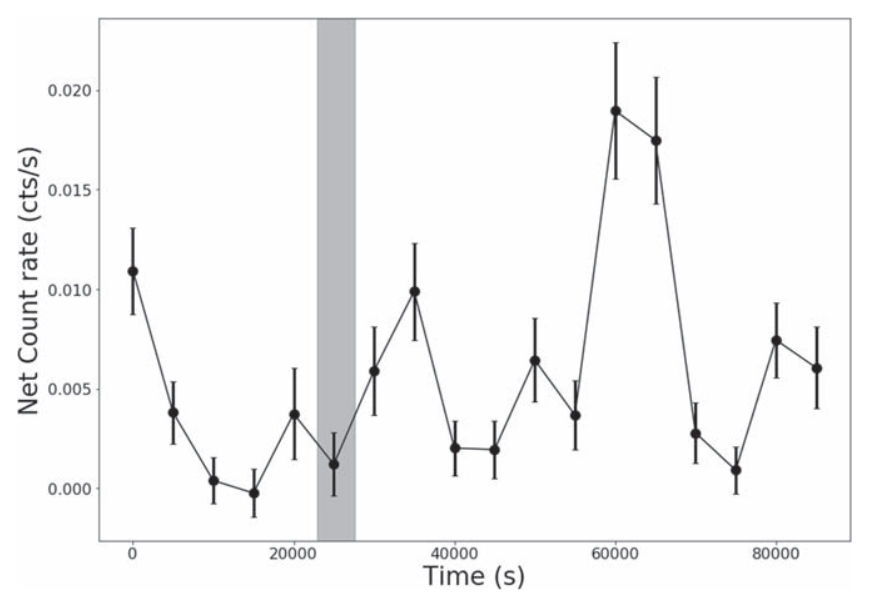

Figure 6. NuSTAR 3-20 keV averaged (over the FPMA and FPMB detectors) net count rate light curve with a $5 \mathrm{ks}$ binning. The source was variable on $\sim 5 \mathrm{ks}$ timescales during this observation, including a period of flux enhancement lasting $\sim 10 \mathrm{ks}$. The gray band shows the time and duration of the joint Swift-XRT observation (observation S4).

(see Figure 6) shows evidence of a flux enhancement lasting $\sim 10 \mathrm{ks}$. To test the significance of this variability we have fit a constant value to the light curve. The best-fit constant has a value of $3.0 \pm 0.9 \times 10^{-3}$ counts $\mathrm{s}^{-1}$ with a $\chi^{2}=93.6$ for 17 degrees of freedom, implying that the source is variable at a $\gtrsim 6.5 \sigma$ level. The $500 \mathrm{~s}$ binned light curve of the period of flux enhancement (see Figure 7) shows the light curve reached a peak count rate of $\sim 0.03 \mathrm{cts} \mathrm{s}^{-1}$. This corresponds to a peak flux of $\sim 1.5 \times 10^{-12} \mathrm{erg} \mathrm{cm}^{-2} \mathrm{~s}^{-1}$, assuming the best-fit power-law model (i.e., $\Gamma=1.2$ and $N_{\mathrm{H}}=5.6 \times 10^{22} \mathrm{~cm}^{-2}$ ). This flux is consistent with the flux observed during the Chandra observation, suggesting that the source's light curve varies over timescales of $\sim 1-10 \mathrm{ks}$.

\subsection{Optical/NIR Companion Photometry}

Using the accurate source position provided by Chandra we have confidently identified the multiwavelength counterpart to this source. Only one Gaia source lies within the $\sim 1^{\prime \prime}(2 \sigma)$ positional uncertainty of AX J1949. The source is also detected in optical by Pan-STARRs (Flewelling et al. 2016), in NIR by 2MASS (Skrutskie et al. 2006), and in IR by WISE 


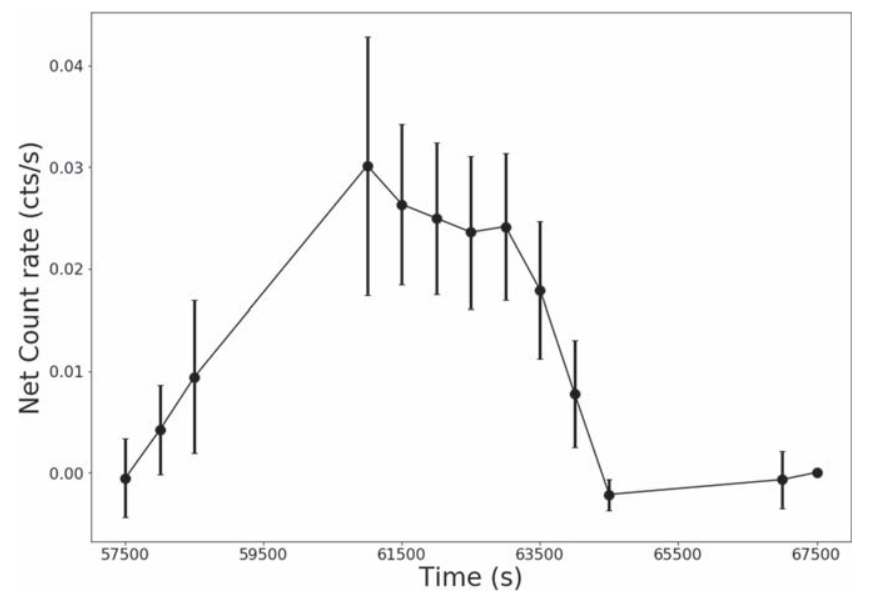

Figure 7. NUSTAR $3-20 \mathrm{keV}$ net count rate light curve with a $500 \mathrm{~s}$ binning during the period of flux enhancement. The source reaches a peak net count rate of $\sim 0.03 \mathrm{cts} \mathrm{s}^{-1}$.

(Cutri et al. 2012). The counterparts multiwavelength magnitudes can be found in Table 3 .

Gaia has also measured the parallax, $\pi=0.081(64)$, and proper motion, $\mu_{\alpha} \cos \delta=-2.37(9), \mu_{\delta}=-5.2(1)$, of $\mathrm{AX}$ J1949's counterpart (Gaia Collaboration et al. 2018). Unfortunately, the Gaia parallax has a large relative error $(\sim 80 \%)$ and, therefore, has a large uncertainty in the inferred distance, $d=6.1_{-1.5}^{+2.3} \mathrm{kpc}$ (Bailer-Jones et al. 2018). We note that this source also has a poorly fit astrometric solution with excess noise, possibly due to its binary nature. Therefore, we do not rely on the Gaia parallax and simply take it as an indicator that the source is at least at a distance of a few kiloparsecs. The large extinction observed in the optical magnitudes of the source also support this claim (see Section 4.1).

\subsection{Optical Spectroscopy}

The optical counterpart of AX J1949 shows $\mathrm{H} \alpha$ emission with $\mathrm{EW} \approx-1.6 \AA$ and FWZI $\approx 500 \mathrm{~km} \mathrm{~s}^{-1}$, and absorption lines of He I $5876 \AA$ and $6678 \AA$ (see Figure 1). The remaining absorption features are diffuse interstellar bands, whose strengths are consistent with the large extinction inferred from the colors of the star (see Section 4.1).

\section{Discussion}

\subsection{Optical/NIR Companion Stellar Type}

The optical spectrum of the companion star shows He I absorption lines, suggesting that the star is hot (i.e., $\gtrsim 10,000 \mathrm{~K}$ ) and, therefore, is likely to be of either $\mathrm{O}$ or B spectral class. Here we use the NOMAD $V$-band photometry in combination with the 2MASS NIR photometry to assess the spectral type, extinction, and distance to this star. The NOMAD $V$-band magnitude and the 2MASS NIR magnitudes can be found in Table 3. To estimate the extinction we use the intrinsic color relationships between the $V$-band and NIR magnitudes provided by Table 2 in Wegner (2014). To recover the intrinsic color relationships of late $\mathrm{O}$ and early to late B-type stars, we need to deredden the source by an $A_{V} \approx 8.5-9.5$, assuming the extinction law of Cardelli et al. (1989). The 3D extinction maps ${ }^{10}$ of

\footnotetext{
10 We use the mwdust python package to examine the dust maps (Bovy et al. 2016).
}

Table 3

Multiwavelength Magnitudes of the Counterpart to AX J19498

\begin{tabular}{|c|c|c|c|c|}
\hline Obs. & $\begin{array}{l}\text { R.A. } \\
\text { (deg.) }\end{array}$ & $\begin{array}{l}\text { Decl. } \\
\text { (deg.) }\end{array}$ & Filter & Mag. \\
\hline \multirow[t]{3}{*}{ Gaia } & $297.480949745(9)$ & $25.56659555(1)$ & $G$ & $13.9860(8)$ \\
\hline & $\cdots$ & $\cdots$ & $B p$ & $16.299(6)$ \\
\hline & $\cdots$ & $\cdots$ & $R p$ & $12.578(3)$ \\
\hline \multirow[t]{5}{*}{$\mathrm{PS}^{\mathrm{a}}$} & $297.480960(2)$ & $25.566612(7)$ & $g$ & $17.516(8)$ \\
\hline & $\cdots$ & $\cdots$ & $r$ & $14.981(4)$ \\
\hline & $\cdots$ & $\cdots$ & $i$ & 13.1990 \\
\hline & $\cdots$ & $\cdots$ & $z$ & 12.1140 \\
\hline & $\cdots$ & $\cdots$ & $y$ & 11.7220 \\
\hline \multirow[t]{3}{*}{ NOMAD } & 297.4809331 & 25.5666150 & $B$ & 18.04 \\
\hline & $\cdots$ & $\cdots$ & $V$ & 16.12 \\
\hline & $\cdots$ & $\cdots$ & $R$ & 14.62 \\
\hline \multirow[t]{3}{*}{ 2MASS } & 297.480989 & 25.566639 & $J$ & $9.90(2)$ \\
\hline & $\cdots$ & $\cdots$ & $H$ & $9.07(2)$ \\
\hline & $\cdots$ & $\cdots$ & $K_{s}$ & $8.63(2)$ \\
\hline \multirow[t]{3}{*}{ WISE } & 297.4809572 & 25.5666135 & $W 1$ & $8.32(2)$ \\
\hline & $\ldots$ & $\ldots$ & $W 2$ & $8.17(2)$ \\
\hline & $\cdots$ & $\cdots$ & $W 3$ & $8.30(3)$ \\
\hline
\end{tabular}

Note.

${ }^{\mathrm{a}}$ Pan-Starrs.

Marshall et al. (2006) suggest a distance of $\approx 7-8 \mathrm{kpc}$ for this range of $A_{V}$.

The reddening and estimated distance to the source can be used to place constraints on its spectral type and luminosity class. In order for the star to have an observed $V$-band magnitude of 16.12 at a distance of $7 \mathrm{kpc}$ with a reddening of $A_{V}=8.5$, it should have an absolute magnitude of $M_{V} \approx-6.6$. The lack of He II absorption features in the optical spectrum suggests that the star is more likely to be of B-type rather than O-type. By comparing the absolute magnitude to Table 7 in Wegner (2006) we find that the star is most likely to be of the Ia luminosity class. Furthermore, the strong He I absorption lines indicate that the star is an early B-type star. However, we mention one caveat, which is that while the lower luminosity classes are not bright enough to be consistent with the distance/reddening to the source, there is a large uncertainty in some of the absolute magnitudes for different luminosity classes (e.g., luminosity class Ib, II; Wegner 2006).

Lastly, the optical spectrum of the star shows an $\mathrm{H} \alpha$ emission line, which is seen in both $\mathrm{Be}$, and supergiant type stars. This line has been observed in a number of similar systems and is often variable in shape and intensity, sometimes showing a P-Cygni like profile (see, e.g., Negueruela et al. 2006a, 2006b; Pellizza et al. 2006). Unfortunately, we have only obtained a single spectrum and cannot assess the variability of the $\mathrm{H} \alpha$ line. Additionally, it does not appear to be P-Cygni like in this single spectrum.

In comparison, by using optical and NIR photometry, Sguera et al. (2017) found the stellar companion of AX J1949 to be consistent with a B0.5Ia type star at a distance, $d=8.8 \mathrm{kpc}$, and having a reddening, $A_{V}=7.2$. We have also found that the star is consistent with an early B-type type star of the Ia luminosity class. However, our estimates of the reddening and distance differ slightly from Sguera et al. (2017), in that we find a larger reddening and smaller distance. 


\subsection{SFXT Nature}

At an assumed distance of $\sim 7 \mathrm{kpc}$, AX J1949's Chandra and Swift-XRT fluxes (when the source is significantly detected) correspond to luminosities of $\sim 1 \times 10^{34} \mathrm{erg} \mathrm{s}^{-1}$ and $\sim 3-8 \times$ $10^{33} \mathrm{erg} \mathrm{s}^{-1}$, respectively. Furthermore, at harder X-ray energies, AX J1949 has a NuSTAR $3 \sigma$ upper-limit quiescent luminosity $\lesssim 3 \times 10^{33} \mathrm{erg} \mathrm{s}^{-1}$ in the $22-60 \mathrm{keV}$ band, while during its flaring X-ray activity detected by INTEGRAL, it reached a peak luminosity of $\sim 10^{37} \mathrm{erg} \mathrm{s}^{-1}$ in the $22-60 \mathrm{keV}$ band (Sguera et al. 2017). This implies a lower-limit on the dynamical range of the system of $\gtrsim 3000$. The large dynamical range and relatively low persistent $\mathrm{X}$-ray luminosity are typical of SFXTs. Additionally, converting the absorbing column density, $N_{\mathrm{H}}=5.6 \times 10^{22} \mathrm{~cm}^{-2}$, derived from the spectral fits to the X-ray data to $A_{V}$, following the relationship provided by Bahramian et al. (2015), we find $A_{V} \approx 20$. This $A_{V}$ is a factor of 2-2.5 larger than the $A_{V}$ derived from the interstellar absorption to the companion star (see Section 4.1). This suggests that a large fraction of the absorption is intrinsic to the source, which has also been observed in other SFXTs (e.g., XTE J1739-302, AX J1845.0-0433; Negueruela et al. 2006a; Zurita Heras \& Walter 2009). Lastly, the optical counterpart to AX J1949 appears to be an early supergiant B-type star, which has been found in $\sim 40 \%$ of the SFXT systems (see Figure 2 in Sidoli 2017). NIR spectra and additional optical spectra should be undertaken to search for additional absorption features, as well as changes in the $\mathrm{H} \alpha$ line profile to solidify the spectral type.

\section{Summary and Conclusion}

A large number of unidentified INTEGRAL sources showing rapid hard X-ray variability have been classified as SFXTs. We have analyzed Neil Gehrels Swift-XRT, Chandra, and NuSTAR observations of the SFXT candidate AX J1949, which shows variability on kilosecond timescales. The superb angular resolution of Chandra has allowed us to confirm the optical counterpart to AX J1949 and obtain its optical spectrum, which showed an $\mathrm{H} \alpha$ emission line, along with $\mathrm{He}$ I absorption features. The spectrum, coupled with multiwavelength photometry allowed us to place constraints on the reddening, $A_{V}=8.5-9.5$, and distance, $d \approx 7-8 \mathrm{kpc}$, to the source. We find that an early B-type Ia is the most likely spectral type and luminosity class of the star, making AX J1949 a new confirmed member of the SFXT class.

We thank Justin Rupert for obtaining the optical spectrum at MDM. We thank the anonymous referee for providing useful and constructive comments that helped to improve the paper. This work made use of observations obtained at the MDM Observatory, operated by Dartmouth College, Columbia University, Ohio State University, Ohio University, and the University of Michigan. This work made use of data from the NUSTAR mission, a project led by the California Institute of Technology, managed by the Jet Propulsion Laboratory, and funded by the National Aeronautics and Space Administration. We thank the NUSTAR Operations, Software and Calibration teams for support with the execution and analysis of these observations. This research has made use of the NuSTAR Data Analysis Software (NuSTARDAS) jointly developed by the ASI Science Data Center (ASDC, Italy) and the California Institute of Technology (USA). J.H. and J.A.T. acknowledge partial support from NASA through Caltech subcontract CIT44a-1085101. J.A.T. acknowledges partial support from Chandra grant GO8-19030X.

Software: CIAO (v4.10; Fruscione et al. 2006), XSPEC (v12.10.1; Arnaud 1996), NuSTARDAS (v1.8.0), Matplotlib (Hunter 2007), Xselect (v2.4e), XIMAGE (v4.5.1), HEASOFT (v6.25), MWDust (Bovy et al. 2016).

\section{ORCID iDs}

Jeremy Hare (다 https://orcid.org/0000-0002-8548-482X Maïca Clavel (i) https://orcid.org/0000-0003-0724-2742 Farid Rahoui 1 i https://orcid.org/0000-0001-7655-4120 John A. Tomsick (1) https://orcid.org/0000-0001-5506-9855

\section{References}

Arnaud, K. A. 1996, adass V, 101, 17

Bahramian, A., Heinke, C. O., Degenaar, N., et al. 2015, MNRAS, 452, 3475 Bailer-Jones, C. A. L., Rybizki, J., Fouesneau, M., Mantelet, G., \& Andrae, R. 2018, AJ, 156, 58

Barziv, O., Kaper, L., Van Kerkwijk, M. H., et al. 2001, A\&A, 377, 925

Bovy, J., Rix, H.-W., Green, G. M., et al. 2016, ApJ, 818, 130

Bozzo, E., Bhalerao, V., Pradhan, P., et al. 2016, A\&A, 596, A16

Bozzo, E., Falanga, M., \& Stella, L. 2008, ApJ, 683, 1031

Bozzo, E., Oskinova, L., Feldmeier, A., \& Falanga, M. 2016, A\&A, 589, A102

Buccheri, R., Bennett, K., Bignami, G. F., et al. 1983, A\&A, 128, 245

Burrows, D. N., Hill, J. E., Nousek, J. A., et al. 2005, SSRv, 120, 165

Cardelli, J. A., Clayton, G. C., \& Mathis, J. S. 1989, ApJ, 345, 245

Cash, W. 1979, ApJ, 228, 939

Chaty, S. 2011, in ASP Conf. Ser. 447, Evolution of Compact Binaries, ed. L. Schmidtobreick, M. R. Schreiber, \& C. Tappert (San Francisco, CA: ASP), 29

Chaty, S. 2015, arXiv:1510.07681

Chaty, S., Rahoui, F., Foellmi, C., et al. 2008, A\&A, 484, 783

Cutri, R. M., Wright, E. L., Conrow, T., et al. 2012, yCat, 2311, 0

Ducci, L., Romano, P., Esposito, P., et al. 2013, A\&A, 556, A72

Ducci, L., Sidoli, L., Mereghetti, S., Paizis, A., \& Romano, P. 2009, MNRAS, 398, 2152

Evans, P. A., Beardmore, A. P., Page, K. L., et al. 2009, MNRAS, 397, 1177

Filliatre, P., \& Chaty, S. 2004, ApJ, 616, 469

Flewelling, H. A., Magnier, E. A., Chambers, K. C., et al. 2016, arXiv:1612. 05243

Fruscione, A., McDowell, J. C., Allen, G. E., et al. 2006, Proc. SPIE, 6270, $62701 \mathrm{~V}$

Gaia Collaboration, Brown, A. G. A., Vallenari, A., et al. 2018, A\&A, 616, A1

Garmire, G. P., Bautz, M. W., Ford, P. G., Nousek, J. A., \& Ricker, G. R., Jr. 2003, Proc. SPIE, 4851, 28

Gehrels, N., Chincarini, G., Giommi, P., et al. 2004, ApJ, 611, 1005

Goossens, M. E., Bird, A. J., Hill, A. B., Sguera, V., \& Drave, S. P. 2019, MNRAS, 485, 286

Grebenev, S. A., \& Sunyaev, R. A. 2007, AstL, 33, 149

Harrison, F. A., Craig, W. W., Christensen, F. E., et al. 2013, ApJ, 770, 103

Hunter, J. D. 2007, CSE, 9, 90

in't Zand, J. J. M. 2005, A\&A, 441, L1

Kim, M., Kim, D.-W., Wilkes, B. J., et al. 2007, ApJS, 169, 401

Krivonos, R. A., Tsygankov, S. S., Mereminskiy, I. A., et al. 2017, MNRAS, 470,512

Lorenzo, J., Negueruela, I., \& Norton, A. J. 2010, in ASP Conf. Ser. 422, High Energy Phenomena in Massive Stars, ed. J. Martí, P. L. Luque-Escamilla, \& J. A. Combi (San Francisco, CA: ASP), 259

Marshall, D. J., Robin, A. C., Reylé, C., Schultheis, M., \& Picaud, S. 2006, A\&A, 453, 635

Matt, G., \& Guainazzi, M. 2003, MNRAS, 341, L13

Negueruela, I., Smith, D. M., Harrison, T. E., \& Torrejón, J. M. 2006a, ApJ, 638, 982

Negueruela, I., Smith, D. M., Reig, P., Chaty, S., \& Torrejón, J. M. 2006b, in ESA Special Publication 604, The X-ray Universe, ed. A. Wislon (Noordwijk: ESA), 165

Pellizza, L. J., Chaty, S., \& Negueruela, I. 2006, A\&A, 455, 653

Porter, J. M., \& Rivinius, T. 2003, PASP, 115, 1153

Reig, P. 2011, Ap\&SS, 332, 1

Rivinius, T., Carciofi, A. C., \& Martayan, C. 2013, A\&ARv, 21, 69 
Romano, P., Bozzo, E., Mangano, V., et al. 2015, A\&A, 576, L4

Romano, P., Ducci, L., Mangano, V., et al. 2014, A\&A, 568, A55

Romano, P., Mangano, V., Cusumano, G., et al. 2011, MNRAS, 412, L30

Romano, P., Sidoli, L., Cusumano, G., et al. 2009, MNRAS, 399, 2021

Sguera, V., Barlow, E. J., Bird, A. J., et al. 2005, A\&A, 444, 221

Sguera, V., Bassani, L., Landi, R., et al. 2008, A\&A, 487, 619

Sguera, V., Bazzano, A., \& Sidoli, L. 2015, ATel, 8250

Sguera, V., Drave, S. P., Bird, A. J., et al. 2011, MNRAS, 417, 573

Sguera, V., Sidoli, L., Paizis, A., et al. 2017, MNRAS, 469, 3901

Shakura, N., Postnov, K., Kochetkova, A., \& Hjalmarsdotter, L. 2012 MNRAS, 420, 216

Sidoli, L. 2013, arXiv:1301.7574

Sidoli, L. 2017, in Proc. XII Multifrequency Behaviour of High Energy Cosmic Sources Workshop (Trieste: SISSA), 52
Sidoli, L., \& Paizis, A. 2018, MNRAS, 481, 2779

Sidoli, L., Romano, P., Ducci, L., et al. 2009, MNRAS, 397, 1528

Sidoli, L., Tiengo, A., Paizis, A., et al. 2017, ApJ, 838, 133

Skrutskie, M. F., Cutri, R. M., Stiening, R., et al. 2006, AJ, 131, 1163

Sugizaki, M., Mitsuda, K., Kaneda, H., et al. 2001, ApJS, 134, 77

Walter, R., Lutovinov, A. A., Bozzo, E., \& Tsygankov, S. S. 2015, A\&ARv, 23, 2

Walter, R., Rodriguez, J., Foschini, L., et al. 2003, A\&A, 411, L427

Walter, R., \& Zurita Heras, J. 2007, A\&A, 476, 335

Wegner, W. 2006, MNRAS, 371, 185

Wegner, W. 2014, AcA, 64, 261

Wilms, J., Allen, A., \& McCray, R. 2000, ApJ, 542, 914

Winkler, C., Courvoisier, T. J.-L., Di Cocco, G., et al. 2003, A\&A, 411, L1

Zurita Heras, J. A., \& Walter, R. 2009, A\&A, 494, 1013 